\title{
Designing a top cooling system for an electromagnetic calorimeter
}

\author{
Matěj Jeřábek ${ }^{1 *}$, Michal Volf ${ }^{1}$, and Daniel Duda ${ }^{1}$ \\ ${ }^{1}$ University of West Bohemia, Faculty of Mechanical Engineering, Department of Power System \\ Engineering, Univerzitní 22, Pilsen, Czech Republic
}

\begin{abstract}
The article describes a numerical simulation of flow in the cooling system of an electromagnetic calorimeter by analysing the temperature and pressure fields. Two fundamentally different approaches were used to analyse the pressure field - analytical 1D calculation and numerical 3D flow simulation. The article contains a detailed evaluation and description of individual analyses using the commercial software ANSYS 2020 R1.
\end{abstract}

\section{Introduction}

What is matter, where does it come from and what is its structure? These are questions that could be answered in experiments at the new Facility for Antiproton and Ion Research (FAIR), which will be part of the existing Helmholtz Centre for Heavy Ion Research (GSI). The complex includes, among other things, the HESR (High Energy Storage Ring) particle accelerator, which houses the PANDA (AntiProton ANnihilation at DArmstadt) detector.

The purpose of this detector is to conduct physical research on topics related to the strong interaction, exotic states and the structure of hadrons [1]. But why? The purpose is related to a phrase we may all have heard before, namely that the whole is not merely the sum of its parts. For some physical cases this theorem certainly does not hold, but for the proton this theorem takes on its true value, since the sum of the masses of the valence quarks in the proton is less than $2 \%$ of its total mass. The rest of the mass is the result of the kinetic and binding energy between the quarks due to the dynamics of the strong nuclear interaction [2].

In order to collect all the necessary information from antiproton-proton collisions, an EMC (Electro Magnetic Calorimeter) will be built as a part of the PANDA detector. The EMC will be able to provide accurate trajectory reconstruction, energy and momentum measurements and very efficient identification of the charged particles. The electromagnetic calorimeter works on the principle of scintillation, i.e., the radiation emitted by the particle collisions reacts with a scintillator that produces a series of flashes of different intensities [3]. These flashes are captured by electronics and transformed into a digital signal. The EMC uses hundreds of cube-shaped scintillators made of $\mathrm{PbWO} 4$, whose scintillation properties are strongly dependent on temperature. Since the light yield from the scintillator crystals increases with decreasing temperature, it is essential to keep these crystals at very low temperatures. In addition, the noise produced by the amplification and subtraction technique

* Corresponding author: ierabeekm@gmail.com 
also decreases with decreasing temperature. Along with the flash intensity, the decay time also varies with temperature [3]. Thus, efforts are made to keep the temperature of the crystals as stable as possible.

This paper briefly describes the design of a cooling system that would allow low crystal temperatures to be maintained, and homogeneous distribution and stability of the temperature over all the crystals while the detector is in operation. Furthermore, the paper will describe the analysis of the effect of the pressure drop of the whole cooling system, from which the design and requirements for the main pump of the system are then derived. Two approaches have been used for the pressure drop analysis, namely an analytical 1D calculation and then a numerical 3D simulation, where the entire geometry of the cooling system is used for the numerical simulation, but without other components occurring in the calorimeter (crystals, segments, etc.). Only a part of the calorimeter geometry was selected for the temperature field analyses, but it was modelled in great detail.

\section{Description of the panda detector}

As already mentioned, HESR is one of the accelerators located in the newly built FAIR complex. Its main components are the PANDA detector and the Koala detector which is used to measure the differential cross section of antiproton-proton elastic scattering [2]. The PANDA detector is composed of several large systems, which can be seen in Fig. 1 [2]. However, in this article we will focus only on the calorimeter of which the proposed cooling system will be a part.

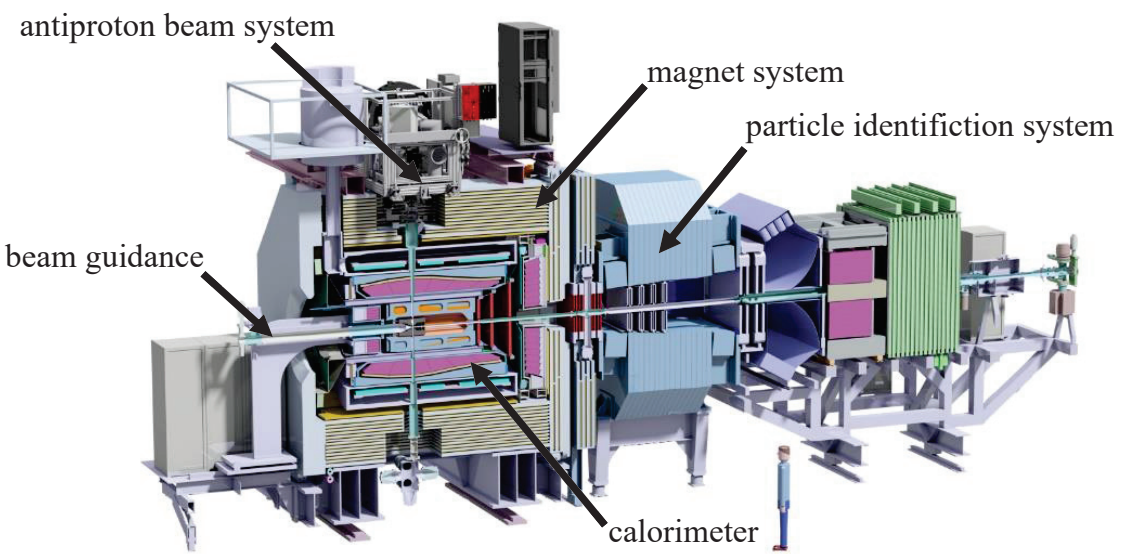

Fig. 1. PANDA detector model [2]

\subsection{Description of the electromagnetic calorimeter}

The calorimeter itself, see Fig. 2a, is made up of 16 segments assembled in the shape of a cylinder. Each segment contains 710 scintillation detectors (blue crystals) rotated to a common point where the particle collisions take place. In Fig. 2a we can also see the crystals located at the back of the calorimeter. However, the cooling system for these (green) crystals needs to be designed separately, as different requirements apply to it. 


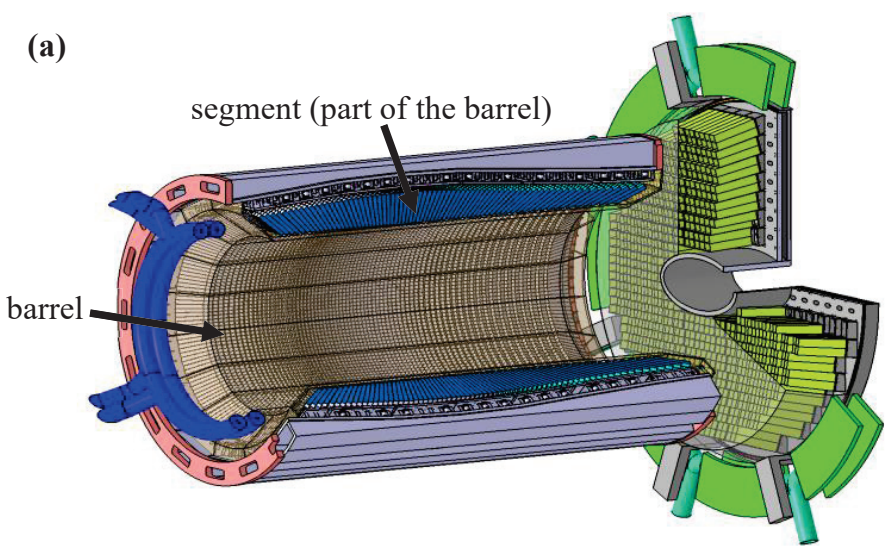

(b)

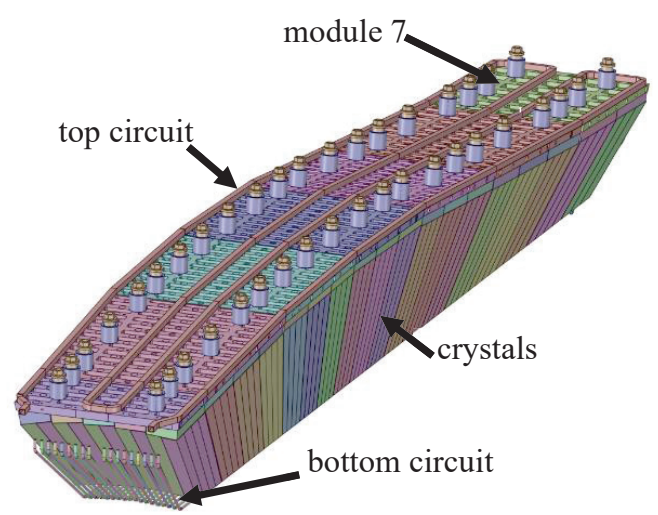

Fig. 2. Top (a): Electromagnetic calorimeter model [4]. Bottom (b): Segment detail

In Fig. 2b we can see the segment without covers and measuring devices, which is divided into seven colour-coded modules. It should be pointed out that the individual modules differ in the inclination of the crystals and also in their number. For subsequent analyses, it is also important to mention that the last module, number 7, has revolving tubes of cooling circuits and therefore it can be assumed that there will be a slightly more intense heat transfer than the other modules.

In addition, a preliminary design of the cooling circuits can be seen in Fig. 2b. It can be seen that each segment will have an upper and a lower cooling circuit.

\section{Cooling system overview}

The primary task of the cooling system is to maintain a very low, stable temperature and homogeneous temperature distribution over all the crystals.

The cooling capacity requirements of the system are determined by the heat sources present in the calorimeter segments. These sources are the measuring or sensing electronics chips, which can be seen in Fig. 3a, and the bus cables, see Fig. 3b. As an additional source of heat, it is necessary to consider the heat that enters the measuring compartment via conduction from the surroundings.

As mentioned, the segments have two separate cooling circuits. Therefore, when designing the cooling system, two separate cooling systems will have to be designed. The upper refrigeration system will consist of the upper refrigeration circuit, as well as the 
distribution piping that will take care of the refrigerant supply and discharge from the upper refrigeration circuits of each segment. The lower cooling system will consist of the same components as the upper cooling system, except that it will supply and discharge refrigerant from the lower cooling circuits. The second main task is to design the cooling systems depending on the total pressure drop, which will ultimately influence the selection of the main pump of the system. The cooling system is schematically shown in Fig. 3c.

(a)

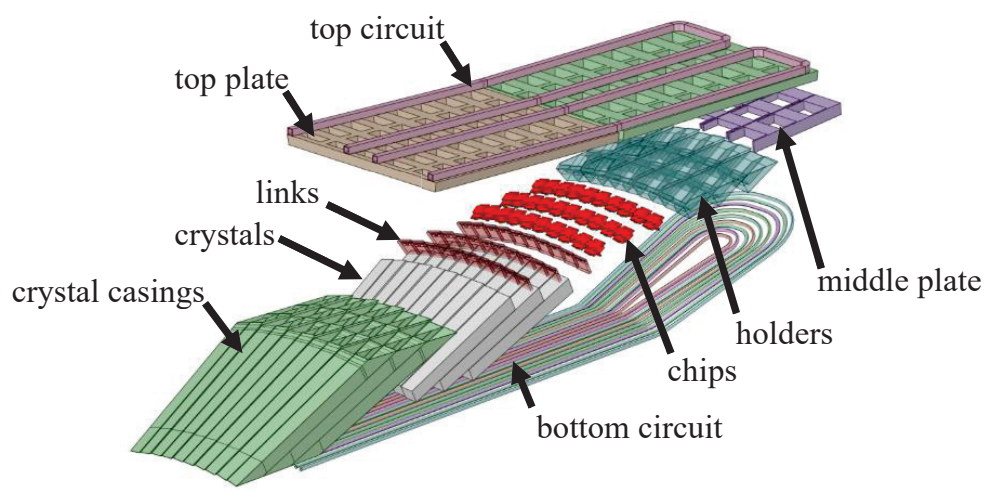

(b)
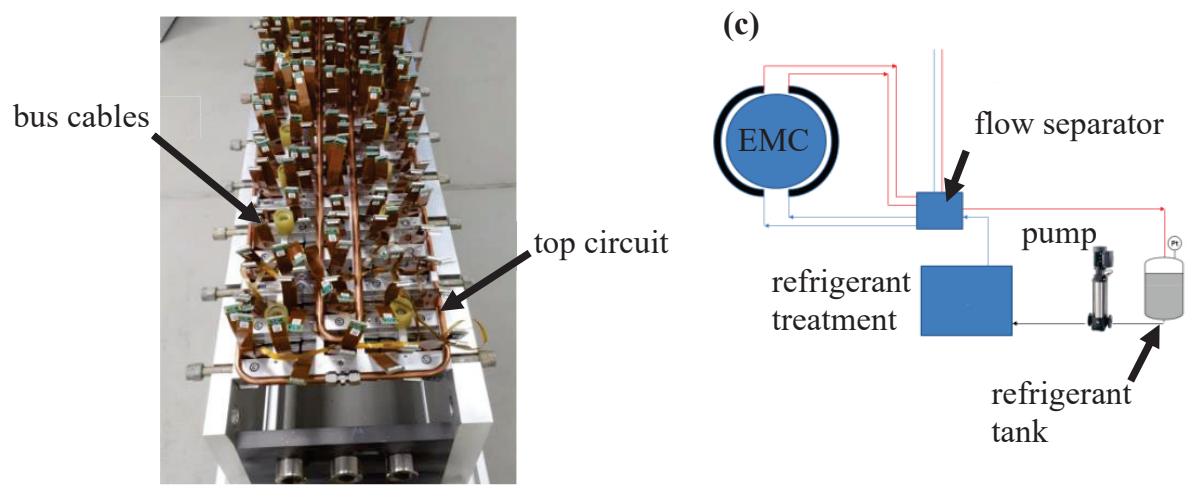

Fig. 3. Top (a): Parts of the module 7. Bottom (b): Preassembled slice [3]. Bottom (c): Cooling system schematics [5]

\subsection{Cooling system requirements}

In terms of temperature field distribution, the requirements are as follows. The operating range of temperatures in which the crystals must move while the calorimeter is running is in the region $-30{ }^{\circ} \mathrm{C}$ to $-20{ }^{\circ} \mathrm{C}$. The stability and homogeneity of the temperature field of the crystals is then determined by the requirement that the maximum temperature difference of all the crystals must be within $1{ }^{\circ} \mathrm{C}$.

In terms of pressure drop, the requirements for the cooling systems are specified in such a way that the pressure drop of the two cooling systems, i.e., the cooling system as a whole, should not exceed 1 bar at the required coolant flow rate of $2.78 \mathrm{~kg} / \mathrm{s}$. This condition follows from the requirement for the pump performance and operating costs. A summary of the initial requirements for the whole cooling system can be found in Table 1. 
Table 1. Summary of requirements [5]

\begin{tabular}{|c|c|c|c|}
\hline $\begin{array}{c}\mathbf{t} \\
{\left[{ }^{\circ} \mathbf{C}\right]}\end{array}$ & $\begin{array}{c}\Delta \mathbf{t} \\
{\left[{ }^{\circ} \mathbf{C}\right]}\end{array}$ & $\begin{array}{c}\Delta \mathbf{p} \\
{[\mathbf{b a r}]}\end{array}$ & $\begin{array}{c}\mathbf{m} \\
{[\mathbf{k g} / \mathbf{s}]}\end{array}$ \\
\hline-30 to -20 & $\leq 1$ & $\leq 1$ & $\leq 2.78$ \\
\hline
\end{tabular}

\subsection{Cooling system working medium}

In order to meet the above requirements, both systems will operate with a 40/60 mix of water and methanol as the coolant. This type of refrigerant is specified by the client, however other mixtures such as water and ethanol could also be considered. The following limiting criteria apply to all types of refrigerants. The refrigerant must maintain a liquid phase even at the very low operating temperatures mentioned. Furthermore, the viscosity of the refrigerant also plays a role in the selection of the refrigerant, as this will ultimately affect the resulting pressure drop of the system, i.e., as the viscosity of the medium increases, the pressure drop increases. Finally, health risks, price, flammability, etc. also play a role in the selection of the refrigerant. The material properties of the refrigerant used are shown in Table 2.

Table 2. Material properties of the refrigerant [5]

\begin{tabular}{|c|c|c|c|c|c|}
\hline Medium & $\begin{array}{c}\mathbf{c}_{\mathbf{p}} \\
{[\mathbf{J} /(\mathbf{k g} \cdot \mathbf{K})]}\end{array}$ & $\begin{array}{c}\mathbf{\rho} \\
{\left[\mathbf{k g} / \mathbf{m}^{3}\right]}\end{array}$ & $\begin{array}{c}\boldsymbol{\mu} \\
{[\mathbf{P a} \cdot \mathbf{s}]}\end{array}$ & $\begin{array}{c}\boldsymbol{\lambda} \\
{[\mathbf{W} / \mathbf{m} \cdot \mathbf{K}]}\end{array}$ & $\begin{array}{c}\mathbf{M} \\
{[\mathbf{k g} / \mathbf{m o l}]}\end{array}$ \\
\hline $\begin{array}{c}\text { water/methanol } \\
(40 / 60)\end{array}$ & 3151 & 930 & $7.7 \mathrm{e}^{-3}$ & 0.341 & 26.5 \\
\hline
\end{tabular}

Where $c_{p}$ is the specific heat capacity of the medium, $\rho$ is the density of the medium, $\mu$ is the dynamic viscosity, $\lambda$ is the thermal conductivity coefficient and $M$ is the molar mass.

\subsection{Cooling system design methodology}

The requirements meant that we approached the design of the cooling system from two perspectives. First of all, the design was based on a numerical simulation performed by researchers at UWB [3], where the bottom circuit was included in the initial simulation of the temperature field and the heat transfer between the individual cooling circuits and crystals was evaluated using this mathematical model.

Secondly, the paper deals with the design of the distribution piping and the implication of this piping in the preliminary design of the cooling circuits, so as to create a complete cooling system that will ensure the transport of the medium to the cooling circuits and then its drainage back to the tank. The system was analysed using two different computational procedures. Firstly, a 3D simulation was performed using CFD (Computational Fluid Dynamics) on a realistic pipe. However, due to the difficulty of performing the simulation (long calculation time, computational complexity, etc.), this simulation was not very suitable for analysing the effect of the changes in the design of the system (number of pipes, change of cross-section, change of dimensions, etc.) on the magnitude of the pressure loss. Therefore, a fast and sufficiently accurate 1D computational tool was developed that would allow a rapid analysis of the effect of changing the system geometry on the pressure drop. This 1D tool will be verified by performing a detailed CFD calculation as experimental data is not available. 


\section{Cooling system design in terms of temperature field}

The computational domain for the numerical simulation of the temperature field was primarily based on the study above [3]. At the outset, it should be added that the geometry of the barrel is quite complicated and simulating the entire barrel would be too time consuming, so we tried to greatly simplify the domain for the analysis. Since each of the segments has its own cooling system, i.e., there is only one cooling system with parallel tubes for each section, it is sufficient to simulate the cooling process in only one section i.e., for one segment instead of the whole barrel. As already mentioned, the segment is composed of seven modules. However, simulating all the modules would again prohibitively increase the computational complexity of the entire simulation. Therefore, we tried to simulate only a part of the geometry in such a way that all the phenomena related to the physical description of the problem are captured and the results are then applicable to the whole geometry. This ultimately allows us to significantly reduce computation time and computational requirements.

In the study [3], it was decided to simulate only the last two modules. This is based on the assumption that module 6 is very similar in design and topology to the other modules, and that module 7 has a reversal of the coolant flow and is therefore expected to have a different heat transfer compared to the other modules.

Taking into account the limitations, assumptions, and outputs of the study [3], a new computational domain was constructed to complement the default one with a bottom cooling circuit. The implementation of the new domain into the default domain can be seen in Fig. 4 .

The geometry of the preliminary design of the lower circuit was used to prepare the complementary domain. Thus, 9 circular tubes with an outer diameter of $6 \mathrm{~mm}$ and an inner diameter of $4 \mathrm{~mm}$ were used. Since the heat transfer had to be considered for the temperature field analysis, the geometry of the tubes was kept in its entirety and was additionally supplemented with a domain representing the refrigerant, i.e., the inverse volume of the tubes, and a domain representing the ambient air.

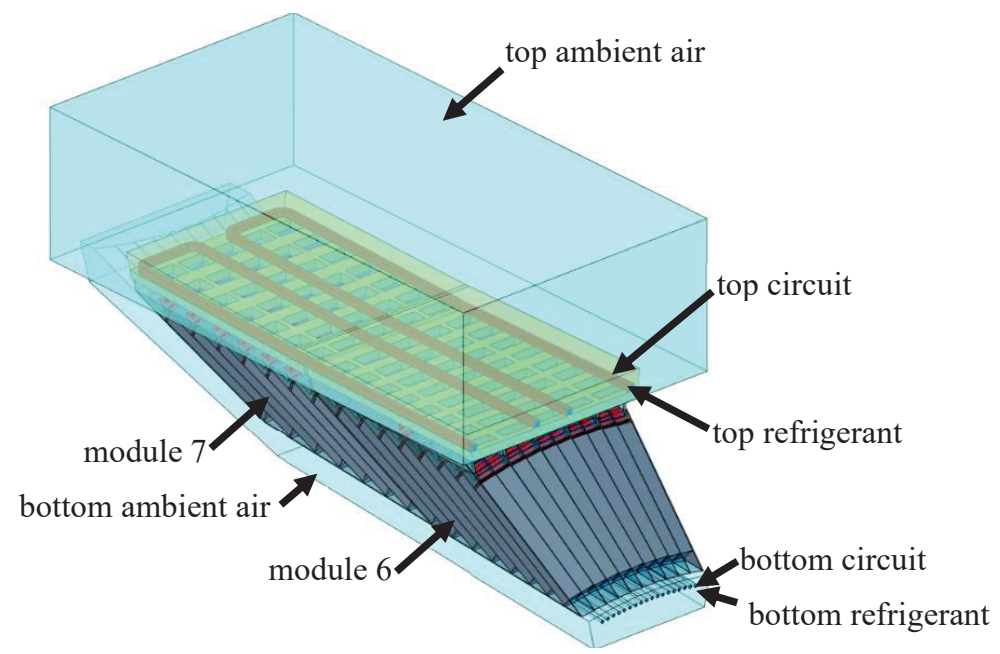

Fig. 4. Computational domain 


\subsection{Simulation setup (temperature field)}

When setting up the numerical simulation, the following inputs were entered into the calculation: the reference pressure of the refrigerant domain was set to 0 bar, so it was not necessary to distinguish between relative and absolute pressure when defining the pressure boundary conditions at the outlet. The turbulence model was chosen to simulate the shedding in the tubes of the circuits by SST k-" $\omega$ " ("Shear Stress Transport") with a turbulence intensity at the inlet of $5 \%$. Gravity was also included in the simulation since the vertical differences in the tubes, especially in the lower circuit, are not negligible. Initial and boundary conditions are also needed to define the CFD simulation. Since this was a stationary simulation, only boundary conditions were used. The inlet coolant boundary condition was set to a temperature of $-30{ }^{\circ} \mathrm{C}$ and a mass flow rate of $0.12 \mathrm{~kg} / \mathrm{s}$. At the outlet of the medium, the outlet pressure condition was set to 1.012 bar, corresponding to the outlet to the atmosphere. The medium used is the mixture of water and methanol (40/60) mentioned earlier, whose material properties are given in Table 2. The heat source in the domain is considered to be the chips of the measuring electronics, whose thermal power is estimated to be $150 \mathrm{~mW}$ [3]. This heat flux will be transferred to the inner walls of the top plate where the processors are expected to be located. The heat flux produced by the bus cables is considered to be $200 \mathrm{~mW}$ [3]. The individual components located in the domain can be seen in Fig 3a. As can be seen (Fig. 3a), the top circuit tubes are located up above the top plate, not around the chips, which are considered as a heat source. Thus, heat transfer is mainly by conduction through a number of components, and thus not only a suitable contact between them must be ensured, but also a lower temperature of the cooling medium to create a sufficient temperature gradient.

A related issue is the need to ensure turbulent flow in the otherwise long and straight sections of tubing where, if laminar flow were to occur, there would be a decrease in heat transfer, thereby increasing the temperature of the refrigerant at the wall. This would ultimately reduce the cooling effect and heat dissipation from the domain.

Finally, the ambient air temperature above the computational domain of the modules is set to $+25^{\circ} \mathrm{C}$. This boundary condition simulates the heat transfer that enters the calorimeter via conduction from the environment. The ambient air temperature below the computational domain of the modules is then set to $-25{ }^{\circ} \mathrm{C}$, simulating the steady state condition that the calorimeter will be in during operation. In other words, the steady state can be understood to mean that in the middle, i.e., in the empty space between the segments, the calorimeter will be cool relative to the crystals. The air in the two domains representing the ambient air is considered as an ideal gas. The reference pressure of the ambient air domains is set to $1 \mathrm{~atm}$. Since the ambient air domain has no inlet or outlet, the pressure level must be defined over the reference pressure.

\subsection{Results (temperature field)}

The results of the stationary CFD simulations of the temperature field show that the addition of the extra domain of the lower cooling circuit has a very positive effect on the resulting temperature field distribution in the crystals. The temperature contours within the computational domain constructed for the numerical temperature field simulations can be seen in Fig. 5.

While in the initial study [3] there was an affected region which meant that the requirement for a homogeneous temperature distribution over all crystals was not met when evaluating the results, this region is no longer present in the completed domain. This change is a major improvement in the temperature field results. From Fig. 5 it can be seen that the temperature difference between all the crystals is now $0.3^{\circ} \mathrm{C}$, and so it can be concluded that 
the requirement for a maximum temperature difference across all the crystals of up to $1{ }^{\circ} \mathrm{C}$ is satisfied. It can also be seen from Fig. 5 that the refrigerant in the upper circuit heats up significantly more than the refrigerant in the lower circuit. This is due to the fact that all the heat sources in the segment are located in the upper part. This implies that the lower circuit may have a lower mass flow rate than the upper circuit. Furthermore, it can be concluded that the preliminary design of the lower circuit is satisfactory for the required crystal cooling.

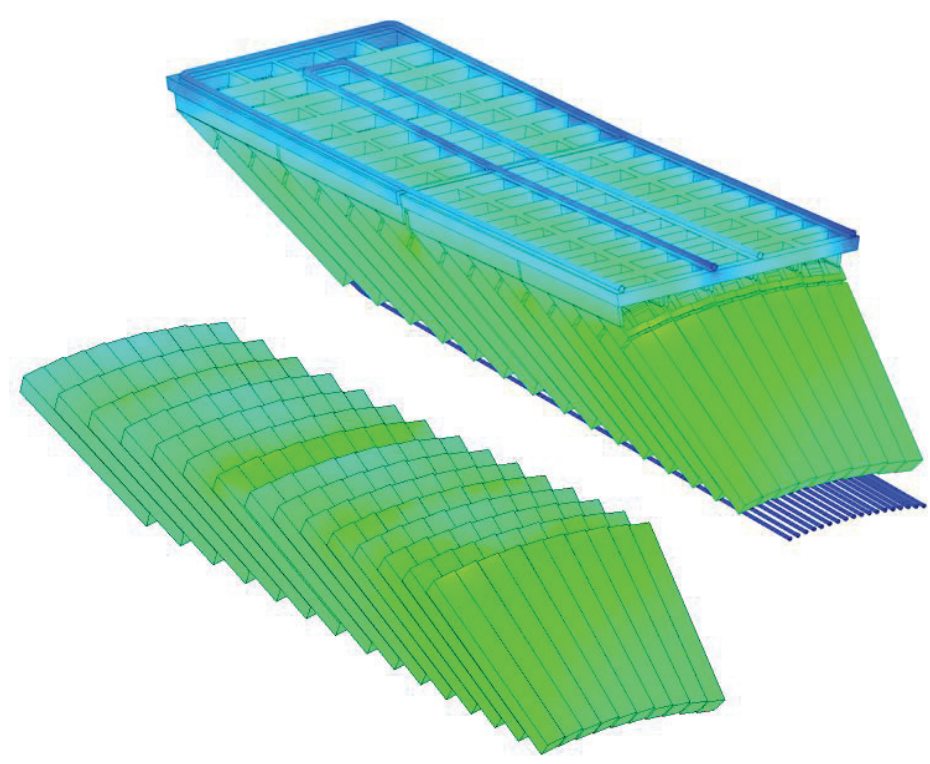

Temperature

Fig. 5. Temperature field distribution 


\section{Cooling system design in terms of pressure loss}

The preparation of the computational domain is different for each of the two design approaches mentioned above. For the pressure loss analysis, the complete domain of the cooling system is considered, i.e., the cooling circuits on all the segments, including the supply and discharge distribution piping. The distribution piping was modelled for the upper and lower circuits. The implementation of the geometry of the distribution piping and individual circuits in the EMC model can be seen in Fig. 6 (a). The systems themselves can be seen in Fig. 6 (b) and (c).

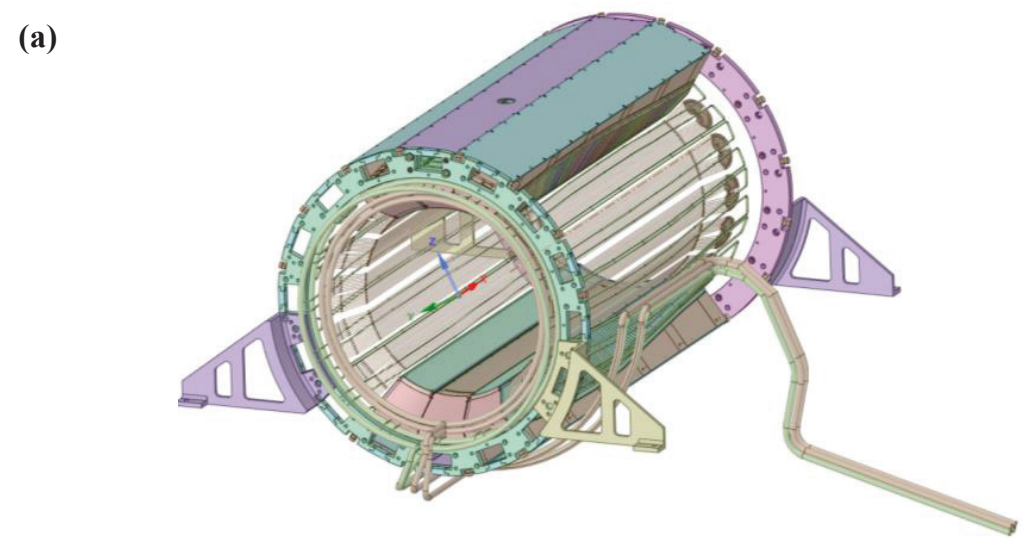

(b)

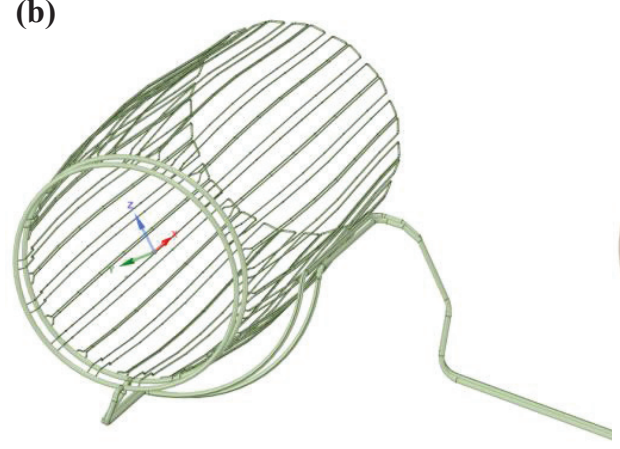

(c)

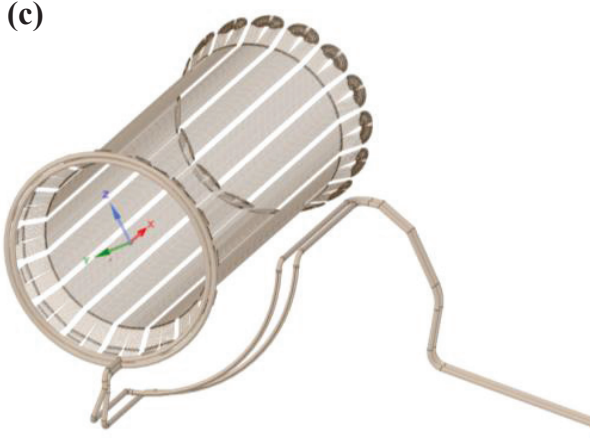

Fig. 6. Top (a): Cooling system in EMC. Bottom (b): Upper cooling system. Bottom (c): Lower cooling system

This setup was further simplified for the CFD simulation by neglecting all the structural components with no influence on the numerical simulation of the pressure drop. It should be noted that the structural components may be neglected because heat transfer is not simulated. Thus we created the upper and lower cooling systems.

As the heat transfer will not be evaluated in the numerical simulation of the flow field, the geometry of the upper and lower systems is further simplified by creating an inverse volume that is occupied by the cooling medium, i.e., neglecting the material of the tube structure. 


\subsection{Computational mesh}

In order to assess the independence of the CFD simulation on the computational network, the network needs to be verified first. A domain consisting of one simplified upper circuit tube and a simplified part of the distribution pipe was prepared. Simplifying the domain reduces the computational demands, reducing the simulation preparation time and thus provides us with the possibility of testing different computational networks and their influence on our results. The verification domain consisting of the components mentioned above can be seen in Fig. 7.

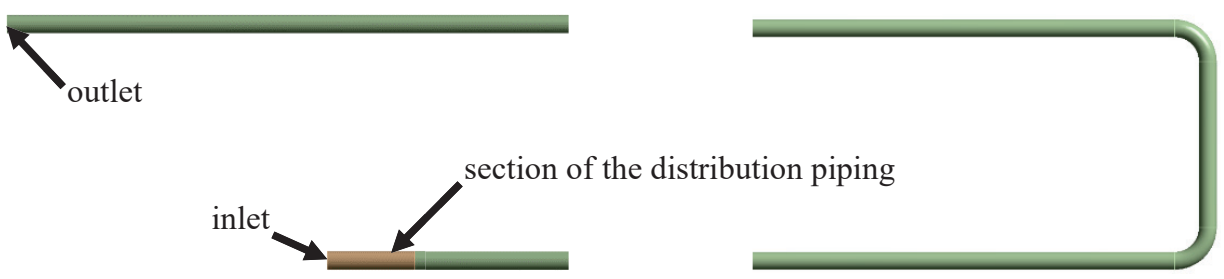

Fig. 7. Verification domain

Although the verification domain contains some geometrical and topological simplifications, it does correspond to the proposed pipeline. The subsequent simulation setup was applied to the verification domain prepared according to the specifications mentioned above. Tetrahedral elements of $3 \mathrm{~mm}$ with a first boundary layer thickness of $0.1 \mathrm{~mm}$ were applied to the part of the domain representing the distribution pipeline.

However, tetrahedral elements are not suitable in terms of stability of the computation and size of the computational mesh, so hexahedral elements with a size of $5 \mathrm{~mm}$ and with an identical first boundary layer thickness were used for the part of the domain representing the pipe. The conformal computational mesh set up according to the specifications can be seen in Fig. 8.

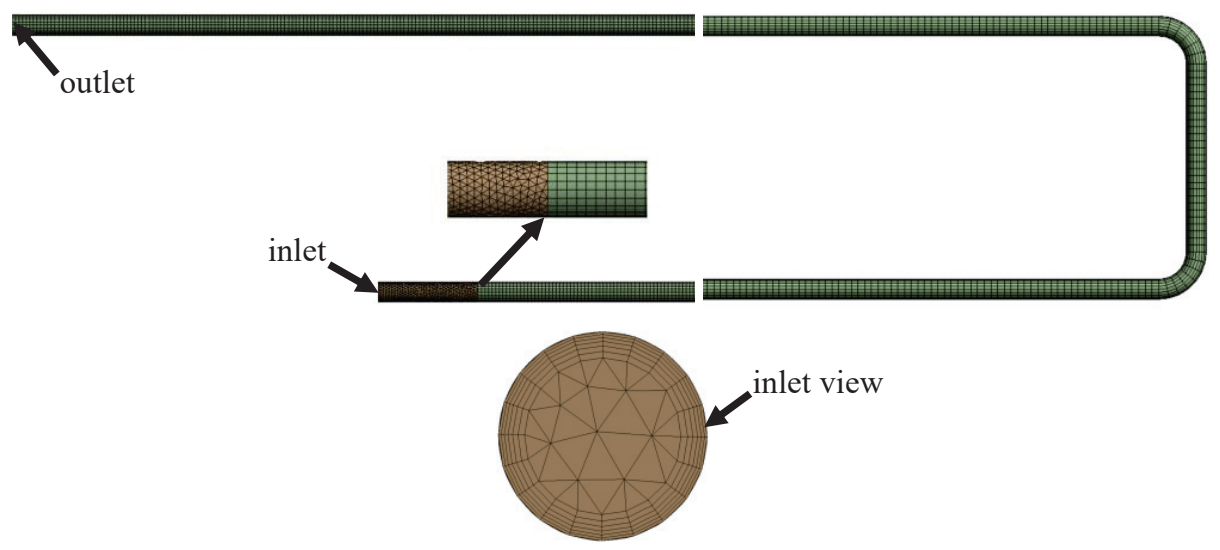

Fig. 8. Mathematical model of the verification domain 


\subsection{Simulation setup (pressure loss)}

When setting up the numerical simulation, the inputs for the refrigerant entered into the calculation were similar to those used in the temperature field simulation. With the difference that the refrigerant mass flow rate was set to $3.84 \mathrm{~m} / \mathrm{s}$, the mass flow rate at the inlet to the cooling system thus set corresponds to a flow rate of $0.12 \mathrm{~kg} / \mathrm{s}$ in one tube of the upper circuit. Another difference in the boundary condition setting is the refrigerant temperature, which has been set to $-25^{\circ} \mathrm{C}$.

\subsection{Results (pressure loss)}

The results from the stationary CFD simulations of the flow field show that the customer's requirements for the total pressure drop of the cooling system were not met. As can be seen from Fig. 9a, the total pressure drop of the upper system is 3.9 bar. For the lower system, in Fig. 9b, the total pressure drop is 5.1 bar.

(a)

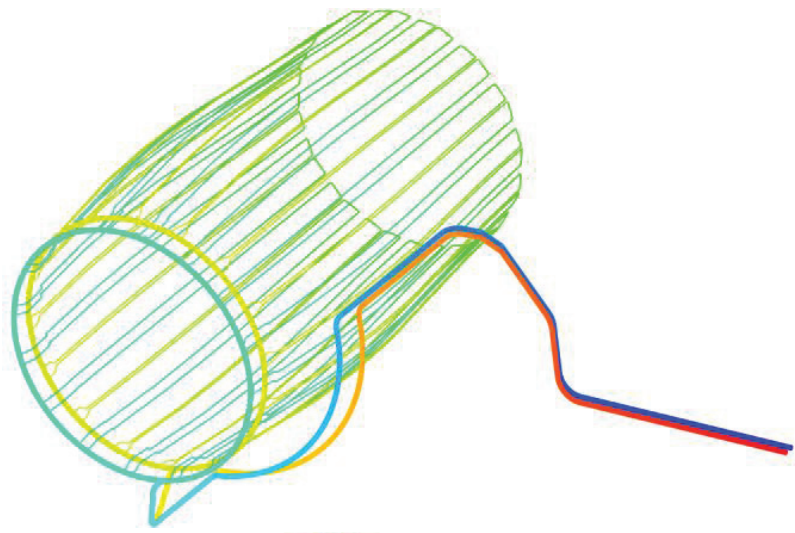

(b)
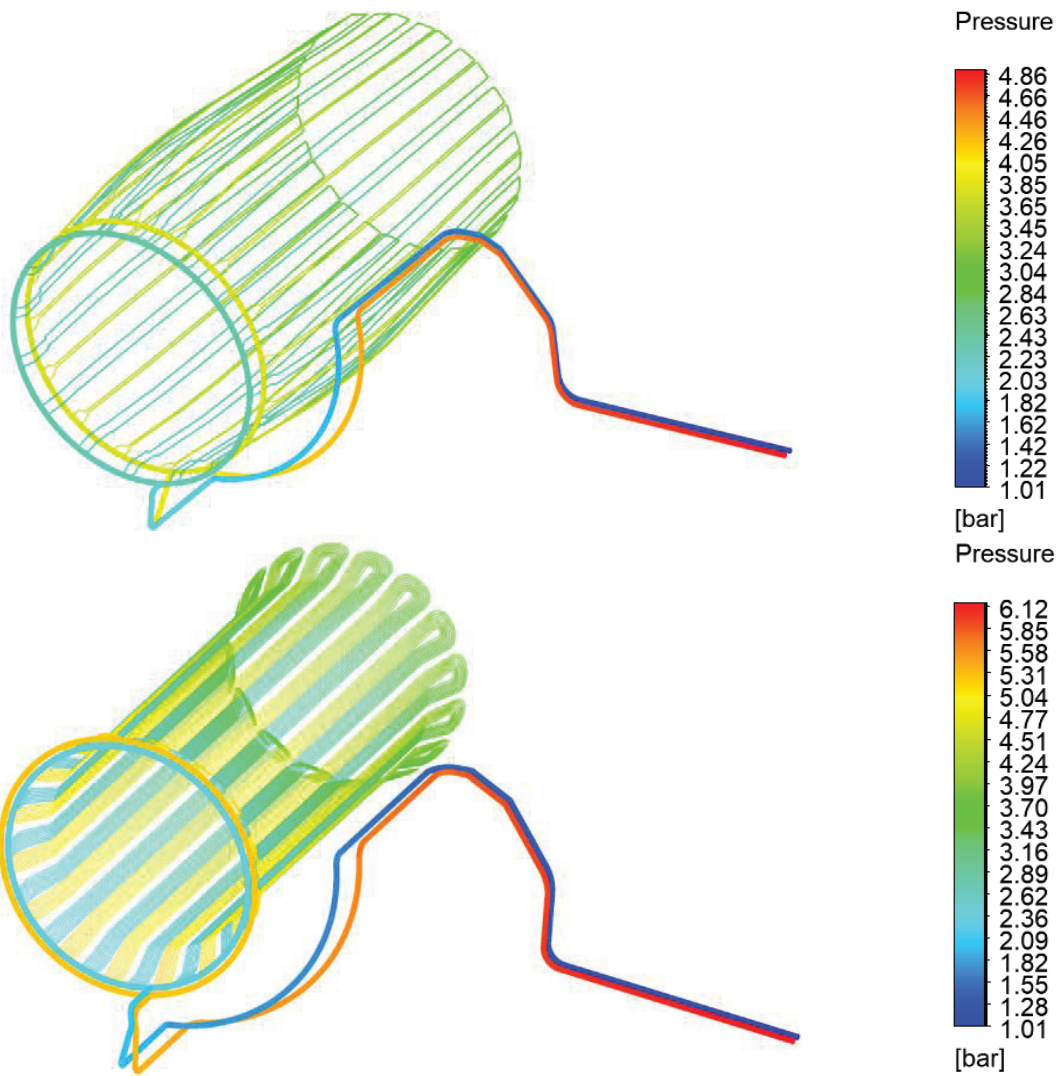

Fig. 9. Top (a): Pressure field of upper cooling system. Bottom (b): Pressure field of bottom cooling system 
In the context of these results, it can be further stated that the customer's required pressure drop of the entire cooling system is an initial conceptual requirement. This consideration is also related to the finding that even the pressure drop of a single tube of the upper cooling circuit alone is not able to meet the requirements for a total pressure drop value of $\Delta \mathrm{p} \leq 1$ bar.

This is because the pressure drop is mostly influenced by the mean velocity in the tubes. This velocity cannot, however, be reduced below the value of $2.5 \mathrm{~m} / \mathrm{s}$ for a single tube, associated with the mass flow rate $0.12 \mathrm{~kg} / \mathrm{s}$, as lowering this velocity would lead to laminar flow in the tubes of the upper circuits, which is unacceptable from the point of view of the required heat transfer.

A compromise must therefore be made between the required heat output of the cooling system and the required pressure drop. This ultimately means that a significantly more powerful pump will be required than was assumed in the initial conceptual design.

\section{Analytical calculation of pressure drop}

The design also includes the creation of a tool to cover the design of the cooling system in terms of pressure losses with sufficient accuracy. For this purpose, we developed a 1D balance calculation based on known analytical formulas for local and frictional pressure losses. A major advantage of this calculation is a significant reduction in computation time, which is considerable for such a large task.

However, the balance calculation involves some simplifications that must be taken into account. Firstly, we neglect the elevation level at which the pipe is located, then we neglect the elevation difference between the inlet and the outlet of the medium. Furthermore, the possibility of different flow patterns in the pipe is not taken into account in the $1 \mathrm{D}$ calculation as we only consider one type of flow along the entire length of the pipe.

\subsection{Cooling system variation analysis}

The analysis was carried out in order to achieve the required (lowest possible) pressure drop of the whole system. It was done so that a reduction in pressure drop could be achieved either by reducing the length of the pipe or by increasing its diameter. This reasoning relates to the assumption that the dominant loss in the system is frictional loss, specifically, the local losses are approximately 10 times smaller for both cooling systems. Therefore, no further consideration will be given to changing the number or angle of the elbows in the distribution piping.

A graph of the dependence of the pressure drop on the diameter and the length of the pipe in the upper system can be seen in Fig. 10. It should be added that since we found that distribution pipe diameters $\leq 0.028 \mathrm{~m}$ have a very high pressure drop value, only diameters larger than this are included in the graph. It should also be added that the shortest distribution pipe length in the graph is $5 \mathrm{~m}$, as the distribution pipe rings themselves are $4.7 \mathrm{~m}$ long. Thus, plotting a graph for lengths of 5-25 m corresponds to a significant number of distribution pipe design variations. The working point of the current design is plotted on the graph. 


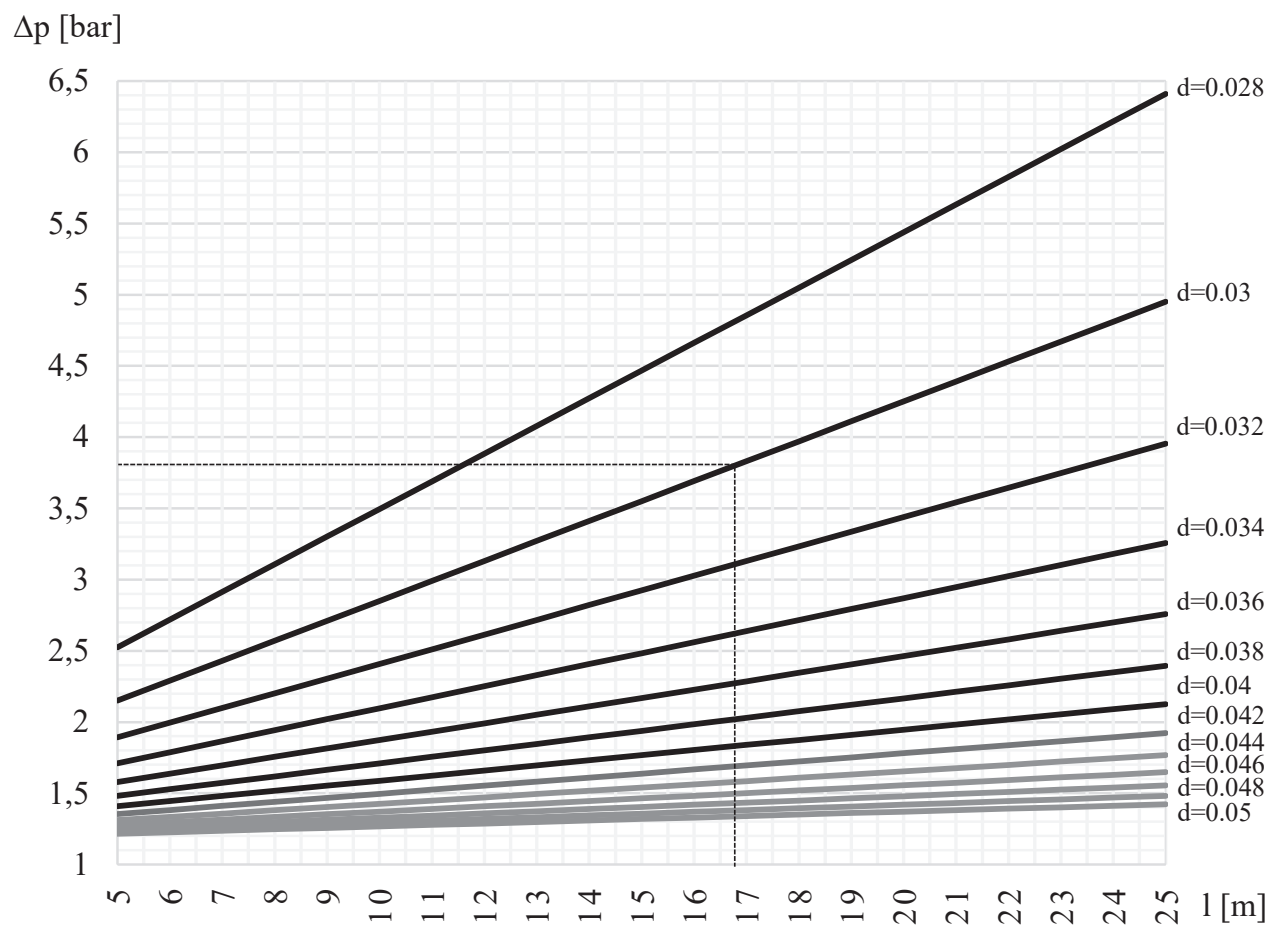

Fig. 10. Dependence of pressure drop on upper cooling system variation

\section{Conclusion}

The main task of this work was to design a cooling system that could keep scintillator crystals at a temperature between $-30{ }^{\circ} \mathrm{C}$ and $-20{ }^{\circ} \mathrm{C}$ during the operation of the calorimeter, so that the difference between the maximum and minimum temperature of all the crystals does not exceed $1{ }^{\circ} \mathrm{C}$. From this point of view, this work was successful, as by adding a bottom cooling system to the initial computational domain, this condition was met, and the maximum temperature difference across all the crystals is now $0.3^{\circ} \mathrm{C}$.

In terms of pressure drop, the customer's requirements have not yet been met, but the requirement that the total pressure drop in the cooling system should not exceed 1 bar appears to be unrealistic, as even the pressure drop of a single tube in the system is approximately 1.13 bar. In view of this fact, the initial requirements have to be reconsidered and modified to take into account the priorities in the system design, which is temperature stability.

Lastly, it should be mentioned that this work also included the creation of a 1D computational tool which, as it turned out, is quite accurately able to cover the design of the cooling system in terms of pressure losses. Although this tool contains some simplifications, it can be used for additional studies dealing with further optimization of the cooling system.

The presented work was financially supported by the student project SGS-2019-021 (Improving the efficiency, reliability and service life of power machines and equipment 5).

Computational resources were supplied by the project "e-Infrastruktura CZ" (e-INFRA CZ ID:90140) supported by the Ministry of Education, Youth and Sports of the Czech Republic. 


\section{References}

1. MESSCHENDORP, Johan. JPS Conference Proceedings. The PANDA Experiment at FAIR-Subatomic Physics with Antiprotons. [Online] 2016. [Citated: 25. 3. 2021.] Available from: https://doi.org/10.7566/JPSCP.13.010016.

2. BELIAS, Tassos a PETERS, Klaus.. PANDA Annual Report. [Online] 2021. [Citated: 5. 1. 2021.] Available from: https://panda.gsi.de/system/files/user_uploads/k.peters/REMGM-2020-002.pdf.

3. VOLF, Michal. AIP Conference Proceedings. CFD Simulation of The Cooling System of a Calorimeter Detector. [Online] 2021. [Citated: 25. 3. 2021.] Available from: https://doi.org/10.1063/5.0041387.

4. Chlazení Elktromagnetického Kalorimetru PANDA s Českou Stopou. Plzeň : ZČU, 2019.

5. ROSIER, P. FOSWIKI. Definition of The Chiller of The Cooling Plant. [Online] 2014. [Citated: 27. 3. 2021.] Available from: https://pandawiki.gsi.de/foswiki/pub/EMC/Cooling/EMC-COOLING-Plant-Chiller-140113.pdf. 